\title{
On the regularization of Lifshitz-type field theories
}

\author{
Alfio Bonanno ${ }^{1,2, a}$, Miok Park ${ }^{3, b}$, Lesław Rachwal ${ }^{4, c}{ }_{(\mathbb{D}}$, Dario Zappalà $^{2, \mathrm{~d}}$ \\ ${ }^{1}$ INAF, Osservatorio Astrofisico di Catania, via S. Sofia 78, 95123 Catania, Italy \\ 2 INFN, Sezione di Catania, via S. Sofia 64, 95123 Catania, Italy \\ ${ }^{3}$ School of Physics, Korea Institute for Advanced Study, Seoul 02455, Republic of Korea \\ ${ }^{4}$ Departamento de Física, ICE, Universidade Federal de Juiz de Fora, Juiz de Fora, MG 36036-900, Brazil
}

Received: 20 October 2020 / Accepted: 12 November 2020 / Published online: 23 November 2020

(C) The Author(s) 2020

\begin{abstract}
We consider Lifshitz-type scalar theories with explicit breaking of the Lorentz symmetry that, in addition, exhibit anisotropic scaling laws near the ultraviolet fixed point. Using the proper time regularization method on the spatial coordinates only, we derive the regularized form of the one-loop effective potential in such theories. We study the main features of the one-loop effective potential and, also, the RG flow of the scale-dependent potential both in the IR and UV regimes. The beta functions for the couplings are derived.
\end{abstract}

\section{Introduction}

Phase transitions associated to Lifshitz points, with their peculiar anisotropic scaling, were introduced and studied long ago, for the first time in [1] and essentially applied to condensed matter problems [2-4]. More recently the presence of Lifshitz points was investigated in the high-energy realm, such as the electromagnetic field theory [5], or the ultraviolet (UV) behavior of scalar fields [6-8], or the more renowned Hořava-Lifshitz formulation of the gravitational theory [9], subsequently generalized to black hole physics (see e.g. $[10,11])$ and cosmology (e.g. $[12,13])$.

The central issue related to the anisotropic Lifshitz points is the non-uniform scaling of the time and space coordinates, that can be summarized as

$t \rightarrow b^{z} t, \quad x^{i} \rightarrow b x^{i}$,

where $b$ is the rescaling parameter and $z$ is the critical scaling exponent. This leads to the following non-uniform scaling

\footnotetext{
a e-mail: alfio.bonanno@inaf.it

be-mail:miokpark@kias.re.kr

c e-mail: grzerach@gmail.com (corresponding author)

de-mail: dario.zappala@ct.infn.it
}

dimensions $[t]_{s}=-z$ and $\left[x^{i}\right]_{s}=-1$ and to the peculiar derivative sector, for instance in the action of a generic scalar field:

$S=\int d^{3} x d t\left(\frac{1}{2} \dot{\phi}^{2}-\frac{1}{2} \alpha^{2}\left(\partial_{i}^{z} \phi\right)^{2}-V\right)$,

where the dot indicates derivative with respect to the time variable, the index $i$ refers to the spatial coordinates, $V(\phi)$ is the potential depending on the field $\phi$ only, and $\alpha$ is a dimensionful constant. Moreover, by the symbol $\partial_{i}^{z}$ we shall understand the $\frac{z}{2}$-th power of the spatially covariant Laplacian operator $\partial_{i}^{2}$. The different scaling dimension of space and time coordinates requires a different number of derivatives with respect to these variables, while the correct dimension is guaranteed by the constant parameter $\alpha$.

The relative weight between the two derivative terms in (2) (which is regulated by the parameter $\alpha$ ) can generate a Lifshitz point that rules the UV physics of the model. In particular, the value of the index $z$, being related to the power of the spatial momentum in the propagator, is crucial to establish the degree of divergence of the various diagrams and therefore the UV structure of the model. On one side, a larger value of $z$ does soften the UV behavior, on the other side it produces a larger violation of the Lorentz symmetry which is instead fully realized when $z=1$. So, for instance, the HořavaLifshitz formulation of gravity requires $z=3$ [9], and one expects that the Renormalization Group (RG) flow towards the infrared (IR) region would modify $z$, so that $z \rightarrow 1$ and in the IR one eventually recovers a Lorentz invariant effective theory.

Incidentally, there is another way to treat all variables on the same footing in order to recover a Lorentz invariant action, that is to require the same non-standard scaling dimension for both time and space variables, which is known as isotropic Lifshitz scaling [1,14]. Thus, for instance, the simplest isotropic Lifshitz scaling is realized for a (Lorentz 
invariant) action containing terms quadratic in the field with four derivatives both in space and time. Isotropic Lifshitz points show interesting properties according to the number of dimensions [15-19], yet maintaining the same number of space and time derivatives of the field.

Actually, in general the explicit violation of Lorentz symmetry has an immediate drawback related to the regularization scheme, which, in general, is constructed accordingly to this symmetry, in such a way to exploit its properties, thus resulting in a simple and elegant procedure. If $z \neq 1$, Lorentz symmetry is lost and one has to choose a suitable regularization scheme to deal with the divergent integrals that appear both in the perturbative computations and also in the development of the RG flow equations within this approach. For instance, in [8], the effective potential of Eq. (2) with $z=2$ is computed by making the most straightforward choice for the UV regulator, i.e. a sharp cutoff on the spatial 3-momentum variable.

However, this sharp cutoff is known not to be suitable in the case of gauge and gravity theories, as it conflicts with the symmetries of the corresponding actions. Therefore, it is convenient to resort to a more flexible regularization method that could preserve such symmetries. To this purpose, we shall consider the proper time regulator [20] that has been widely used both in the computation of the effective potential and of its RG improvements in the case of standard scaling [21-28], and that has been recently revisited in $[29,30]$ and, also, turns out to be more appropriate to treat gauge theories [23]. This kind of regulator was used as well to evaluate the RG flow of a scalar theory in the case of isotropic Lifshitz scaling [15], and we expect that it can be suitably adapted to the anisotropic case. In fact, an approach to the computation of the effective potential similar to the one we shall discuss in this paper is presented in [31], although in our opinion not thoroughly elaborated.

In what follows, we will consider the toy model analyzed in [8], i.e. the action in (2) with $z=2$. In the action, in addition to the full potential $V$ that includes all higher order terms in the field $\phi$ (which, in this framework, has scaling dimension $[\phi]_{s}=1 / 2$ ), we retain the marginally deformed kinetic term only and neglect other renormalizable derivative operators that would pointlessly complicate our analysis. Since the theory naturally splits space and time, we shall deal with them separately.

In Sect. 2, we construct a proper time representation specifically for this case and compute the one-loop effective potential. In Sect. 3, we analyze some details of the renormalized one-loop effective potential, while in Sect. 4 we determine the RG flow that gives access to the $\beta$-functions of the various couplings. Our conclusions are reported in Sect. 5.

\section{Regularization scheme}

As a first step we compute the one-loop effective potential of the action (2) with the tree potential

$V=\frac{m^{2}}{2} \phi^{2}+\sum_{n=1}^{4} \frac{\lambda_{n}}{(2 n+2) !} \phi^{2 n+2}$,

that contains only the relevant (and marginal) powers of the field $\phi$, according to the non-standard scaling dimensions outlined above.

The one-loop computation involves integrals over the four momentum components, but the different scaling of the space and time variables implies the breaking of the full Lorentz symmetry. Therefore, in the loop integrals it is convenient to first perform the integral over $p^{0}$, which resembles the same integral in the standard case, and only later the integral over the spatial momenta $p^{i}$.

In our case of modified kinetic term, to the one-loop accuracy and up to a field independent infinite constant, the quantum effective potential in Minkowski spacetime takes the following form

$$
\begin{aligned}
V_{11} & =-i \hbar \frac{1}{2} \int \frac{d^{4} p}{(2 \pi)^{4}} \ln \frac{\delta^{2} S}{\delta \phi^{2}} \\
& =-i \hbar \frac{1}{2} \int \frac{d^{4} p}{(2 \pi)^{4}} \ln \left[p_{0}^{2}-\alpha^{2}\left(\vec{p}^{2}\right)^{2}-V^{\prime \prime}+i \epsilon\right]
\end{aligned}
$$

where $V^{\prime \prime}$ is the second derivative of the potential with respect to the field $\phi$ and $\epsilon$ is a positive constant which should be sent to zero in the end. After performing the $p^{0}$ integral we are left with the spatial integral

$V_{11}=\frac{1}{2} \int \frac{d^{3} \vec{p}}{(2 \pi)^{3}} \sqrt{\alpha^{2}\left(\vec{p}^{2}\right)^{2}+V^{\prime \prime}}$,

which, formally, is the same result of the standard approach, provided one defines the energy $E$ in this case through the modified dispersion relation $E^{2}=\alpha^{2}\left(\vec{p}^{2}\right)^{2}+V^{\prime \prime}$. Then, on a vanishing background the second derivative of the potential $V^{\prime \prime}$ is a constant and the UV divergences in our problem appear in the resolution of the integral in (5). The latter shows an $O(3)$ symmetry that can be used to integrate over the angular variables, to obtain

$V_{11}=\frac{1}{4 \pi^{2}} \int d p p^{2} \sqrt{\alpha^{2} p^{4}+V^{\prime \prime}}$.

At this point, instead of treating the UV divergences by cutting off the UV modes by means of an upper extremum $\Lambda$ in the integral (6), as done in [8], we resort to the more suitable regularization method known as proper time [20], and adapt it to the specific form of the integral (6). Namely, 
we use the following integral representation of the square root appearing above:

$\int_{0}^{\infty} d s A e^{-s \sqrt{A}}=\sqrt{A}$,

which is valid for any $A>0$. This assumption is clearly satisfied in the case of Euclidean version of the Lifshitz-type model, provided $V^{\prime \prime}>0$, i.e. we are in the symmetric phase. We can take

$A=p^{4}+\alpha^{-2} V^{\prime \prime}$,

where we rescaled for our convenience the whole kinetic term by powers of the dimensionful $\alpha$ parameter. We remind here that we have $[\alpha]=-1$. Moreover, we notice that the dimension of the regulator in the proper time is $[s]=-2$. Then, by combining (7) with (8) and plugging this in the integral (6), we get

$V_{11}=\frac{\alpha}{4 \pi^{2}} \int_{0}^{\infty} d s \int_{0}^{\infty} d p p^{2}\left(p^{4}+\frac{V^{\prime \prime}}{\alpha^{2}}\right) e^{-s \sqrt{p^{4}+\alpha^{-2} V^{\prime \prime}}}$,

which is the proper time representation of the integral for the one-loop potential of our model. We multiplied the integrand by additional power of the $\alpha$ parameter to comply with the energy dimension of the potential (in $d=4$ we have $[V]=$ $\left.\left[V_{11}\right]=4\right)$. It is important to notice that, for the success of the proper time regularization program, the integral over the proper time parameter $s$ must be done at last, not to interfere with the integration over spatial momentum.

The momentum integral in (9) can be done in an analytic compact form, for a general Lifshitz tree-level potential $V=V(\phi)$ taken as a general function of the background scalar field, through a clever change of integration variable. The rough idea is to remove the square root from the exponent and the price is that it (and its derivatives) will appear in the numerator and also in the denominator of the integrand expression. We introduce a dimensionless integration variable $y$ (instead of the dimensionful $p$, with $[p]=1$ ) defined by the equation

$\frac{y^{4} V^{\prime \prime}}{\alpha^{2}}=\frac{V^{\prime \prime}}{\alpha^{2}}+p^{4}$,

where we retain only real positive $p$, so that we can rewrite

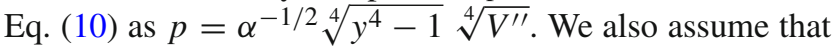
the allowed range for the $y$ variable is $\langle 1,+\infty)$ and that $V^{\prime \prime}$ and $\alpha$ are naturally positive. By inserting into the momentum integral the Jacobian $\mathcal{J}$ of this change of integration variables, $\mathcal{J}=d p / d y=\alpha^{-1 / 2} y^{3} \sqrt[4]{V^{\prime \prime}}\left(y^{4}-1\right)^{-3 / 4}$, we get

$$
\begin{gathered}
\int_{0}^{\infty} d p p^{2} \alpha\left(p^{4}+\frac{V^{\prime \prime}}{\alpha^{2}}\right) e^{-s \sqrt{p^{4}+\alpha^{-2} V^{\prime \prime}}} \\
=\int_{1}^{\infty} d y \frac{y^{7} V^{\prime \prime 7 / 4} \exp \left(-\frac{s y^{2} \sqrt{V^{\prime \prime}}}{\alpha}\right)}{\alpha^{5 / 2} \sqrt[4]{y^{4}-1}} .
\end{gathered}
$$

By recalling that the integral

$F(a)=\int_{1}^{\infty} d y \frac{y^{7} \exp \left(-a y^{2}\right)}{\sqrt[4]{y^{4}-1}}$

is expressible in a compact form through the combination of Gamma, $\Gamma$, and Bessel functions, $I$, as

$F(a)=\frac{\sqrt{\pi} \Gamma\left(\frac{7}{4}\right)\left(3 I_{-\frac{9}{4}}(a)-2 a I_{-\frac{5}{4}}(a)+6 I_{\frac{9}{4}}(a)+2 a I_{\frac{13}{4}}(a)\right)}{3 \sqrt[4]{2} a^{5 / 4}}$,

we write the final results for the one-loop effective potential in the form $\left(a=s \sqrt{V^{\prime \prime}} / \alpha \equiv s v>0\right)$

$$
\begin{aligned}
V_{11}= & \frac{1}{4 \pi^{2}} \int_{0}^{\infty} d s \frac{V^{\prime \prime 7 / 4}}{\alpha^{5 / 2}} F\left(\frac{s \sqrt{V^{\prime \prime}}}{\alpha}\right) \\
= & \frac{v^{9 / 4} \alpha \Gamma\left(\frac{3}{4}\right)}{8 \sqrt[4]{2} \pi^{3 / 2}} \int_{0}^{\infty} d s s^{-5 / 4}\left[\frac{3}{2} I_{-\frac{9}{4}}(s v)\right. \\
& \left.+3 I_{\frac{9}{4}}(s v)+s v\left(I_{\frac{13}{4}}(s v)-I_{-\frac{5}{4}}(s v)\right)\right] .
\end{aligned}
$$

Now, the UV divergence is contained in the proper time $s$ integral and it can be regularized by simply putting a lower cut-off $s_{\mathrm{UV}}$ :

$\int_{0}^{\infty} d s \rightarrow \int_{s_{\mathrm{UV}}}^{\infty} d s=\int_{B k^{-2}}^{\infty} d s$

This cutoff does not act directly on the momentum, but on the proper time $s$, and this allows to bypass the various drawbacks related to the momentum cut-off. Also, in the right hand side of (15) we redefine the cutoff $s_{U V}$ in terms of a (running) scale $k$, by also including a free constant dimensionless parameter $B$, to be adjusted later, namely $s_{\mathrm{UV}}=B k^{-2}$.

The introduction of the cutoff $s_{\mathrm{UV}}$, makes it possible to single out the divergent part (in the limit $s_{\mathrm{UV}} \rightarrow 0$ ) of the potential, which has the structure of a sum of inverse powers of $s_{\mathrm{UV}}$. Then, the simplest renormalization scheme corresponds to the plain subtraction of the divergent terms only, 
Fig. 1 The effective potential at the classical, (upper blue curve) $V$, and one-loop level, (lower red curve) $V_{\text {tot }}=V_{11}+V_{\mathrm{ct}}+V$, plotted for values of the field $\phi$ around the classical minimum at $\phi=0$. A field-independent constant is subtracted in $V_{\text {tot }}$, in order to get $V_{\text {tot }}(\phi=0)=0$. The values of the parameters are $m^{2}=\lambda=1$ and $\alpha=0.1$

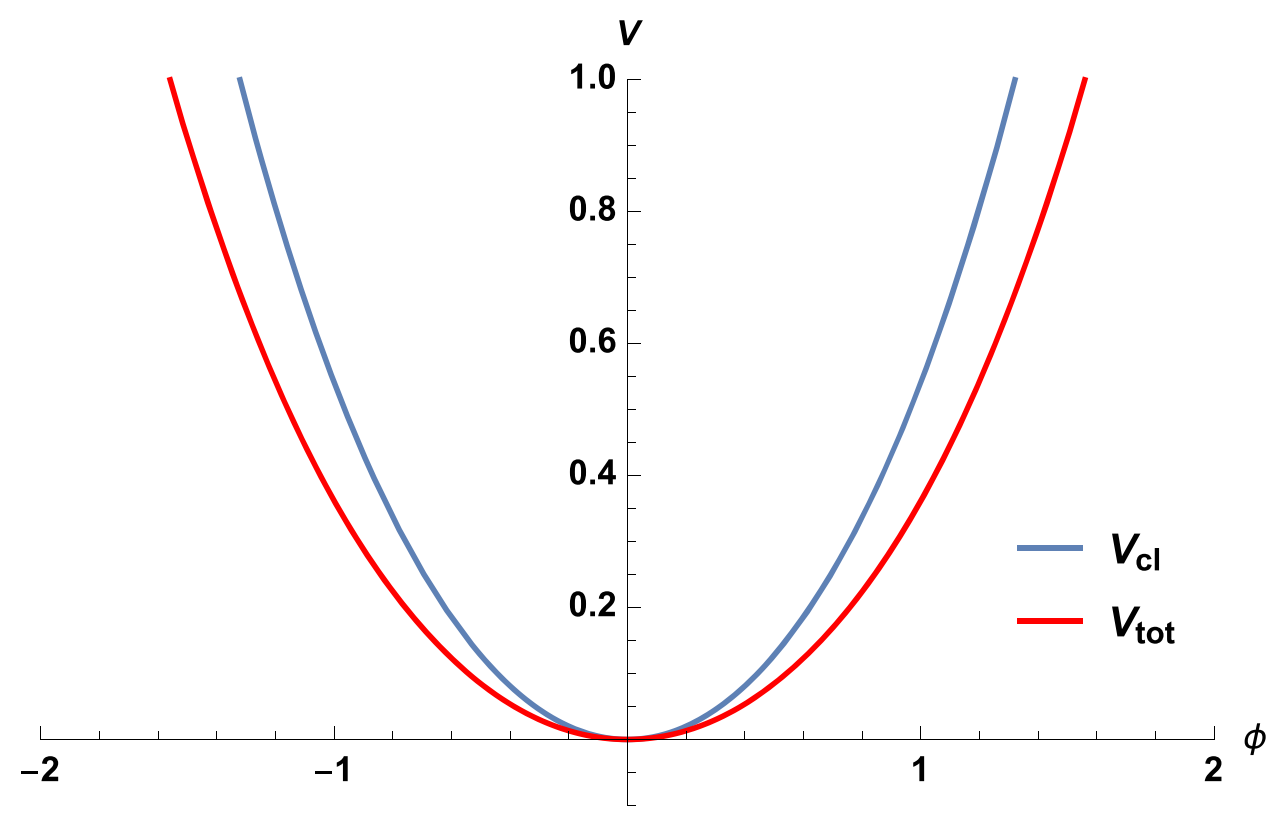

via the inclusion of suitable counterterms. Namely, we perform the following subtraction

$$
\begin{aligned}
V_{11}+V_{\mathrm{ct}}= & \alpha \int_{0}^{\infty} d s \frac{v^{9 / 4} \Gamma\left(\frac{3}{4}\right)}{16 \sqrt[4]{2} \pi^{3 / 2}} s^{-5 / 4}\left[3 I_{-\frac{9}{4}}(s v)+6 I_{\frac{9}{4}}(s v)\right. \\
& \left.+2 s v\left(I_{\frac{13}{4}}(s v)-I_{-\frac{5}{4}}(s v)\right)\right] \\
& -\alpha \int_{0}^{\infty} d s\left(\frac{15}{64 \pi^{3 / 2}} \frac{1}{s^{7 / 2}}+\frac{1}{64 \pi^{3 / 2}} \frac{v^{2}}{s^{3 / 2}}\right) .
\end{aligned}
$$

The difference of integrals in Eq. (16), generated by our specific renormalization scheme, can be performed analytically, and the output is (see also [31])

$V_{11}+V_{\mathrm{ct}}=\frac{\alpha \Gamma\left(-\frac{1}{4}\right) v^{5 / 2}}{64 \sqrt{2} \pi^{3 / 2} \Gamma\left(\frac{9}{4}\right)}$.

\section{Analysis of the effective potential}

The result obtained in Eq. (17) directly yields the full renormalized one-loop effective potential that, in the original variables and after some manipulation of the Gamma functions, reads

$V_{\mathrm{tot}}=V+V_{11}+V_{\mathrm{ct}}=V-\frac{\Gamma\left(\frac{3}{4}\right)^{2}}{10 \pi^{5 / 2} \alpha^{3 / 2}} V^{\prime \prime 5 / 4}$

Clearly, $V_{\text {tot }}$ is real only for field values $\phi$ such that $V^{\prime \prime} \geqslant 0$, and it precisely reproduces Eq. (29) of [8].

Now we show in three elucidatory, numerical examples the comparison of the tree potential

$V=\frac{1}{2} m^{2} \phi^{2}+\frac{\lambda}{4 !} \phi^{4}$ with the renormalized one-loop potential $V_{\text {tot }}$, and we notice that, because of the minus sign in front of the loop contribution in Eq. (18), the quantum corrections to $V$ are negative at any value of the field $\phi$. This is observed in Fig. 1, where $V$ (upper blue curve) and $V_{\text {tot }}$ (lower red curve) are plotted in a typical configuration with no spontaneous symmetry breaking (SSB), $m^{2}=\lambda=1$, and $\alpha=0.1$. (In order to see appreciable differences between the two curves with $m^{2}$ and $\lambda$ set at 1 , it must be $\alpha \ll 1$.)

In addition, for convenience, we subtracted from $V_{\text {tot }}$ its value at $\phi=0$ :

$V_{\text {tot }}(\phi=0)=-\frac{m^{5 / 2} \Gamma\left(\frac{3}{4}\right)^{2}}{10 \pi^{5 / 2} \alpha^{3 / 2}}$,

which, for the choice of the parameters used in Fig. 1, is about $V_{\text {tot }}(\phi=0) \simeq-0.2715$, so that for the red curve one observes $V_{\text {tot }}(\phi=0)=0$.

For a smaller value of $\alpha$ we observe the generation of new SSB minima via quantum corrections, for the same tree-level potential. This is illustrated in the example in Fig. 2 where, as in Fig. $1 m^{2}=\lambda=1$, while $\alpha=0.04$. Again, for the sake of the comparison, $V_{\text {tot }}(\phi=0) \simeq-1.0730$ is subtracted from the one-loop effective potential, so that the two curves coincide at $\phi=0$. Here the full one-loop curve shows two degenerate absolute minima at non-vanishing values of the field $\phi$ (and therefore SSB), while $\phi=0$ turns out to be a local maximum. Other details of the onset of SSB will be discussed below.

Finally, the plot in Fig. 3 compares the tree-level potential specified by $\lambda=1$ and $m^{2}=-0.2$, and therefore displaying SSB at tree level, with the real part of the full one-loop potential at $\alpha=0.1$, which shows even deeper minima at non- 
Fig. 2 The effective potential at the classical, (upper blue curve) $V$, and one-loop level, $V_{\text {tot }}$ (lower red curve). As in Fig. $1, m^{2}=\lambda=1$ and a constant is subtracted from $V_{\text {tot }}$, so that $V_{\text {tot }}(\phi=0)=0$. The value of the remaining parameter is $\alpha=0.04$ and in this case $V_{\text {tot }}$ shows SSB
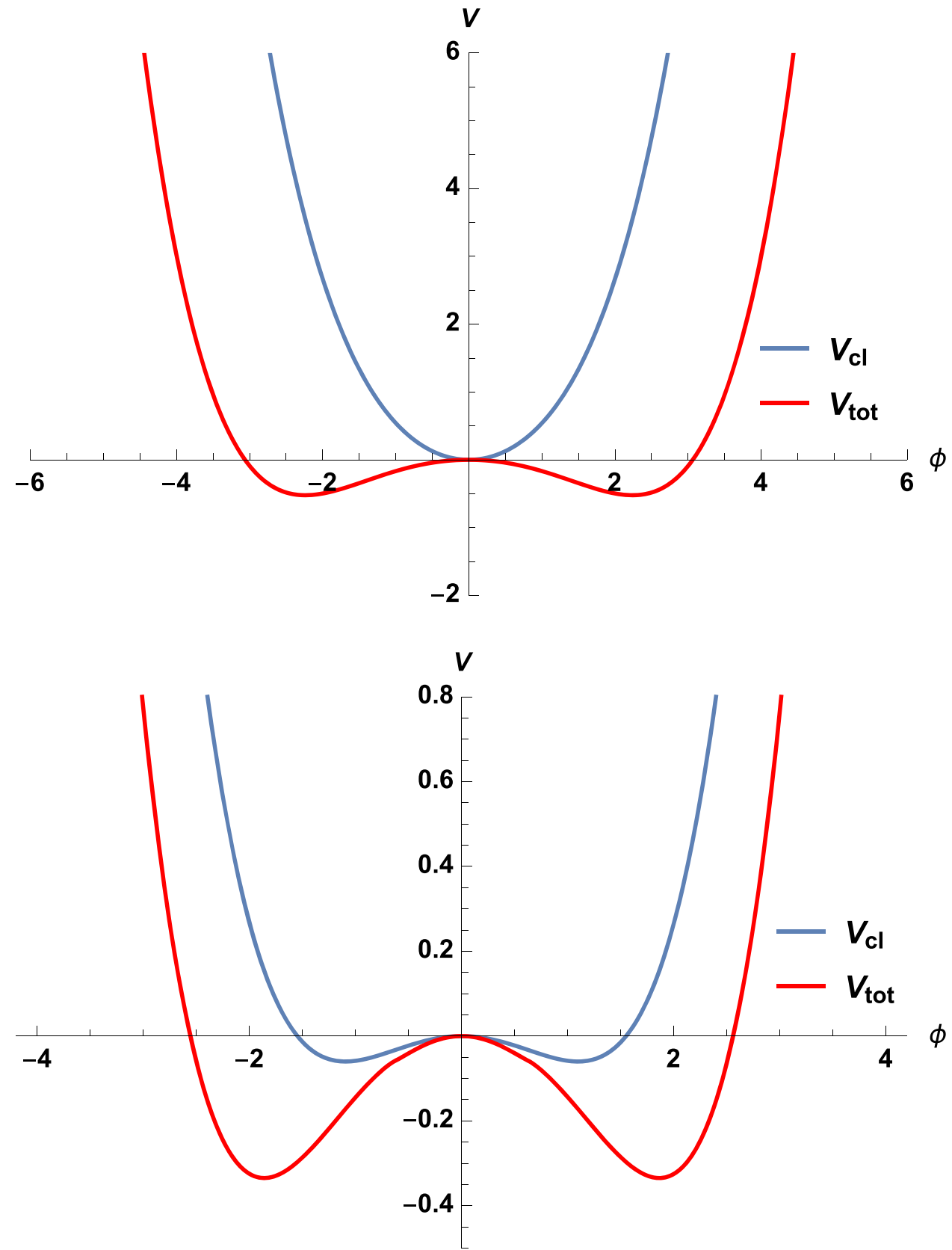

Fig. 3 The real part of effective potential at the classical, (upper blue curve) $V$, and one-loop level, $V_{\text {tot }}$ (lower red curve) with parameters $m^{2}=-0.2, \lambda=1$ and $\alpha=0.1$. The real part of the effective potential at $\phi=0$ is subtracted from $V_{\text {tot }}$ in order to obtain the lower red curve. In this case both curves show SSB vanishing $\phi$. It must be remarked that in this case, with negative $m^{2}$, quantum corrections are real at large values of the field, but become complex for $|\phi|<\sqrt{-2 m^{2} / \lambda}=\sqrt{2 / 5} \approx$ 0.6325 , and therefore, in this region of small field, we plot only the real part of the complex total potential $V_{\text {tot }}$. In addition, even in Fig. 3 the one-loop potential curve is adjusted to have a vanishing effective potential at the origin and this means that we subtracted the real value of Eq. (20), which for our choice of the parameters is approximately 0.0257 .

After having shown in the figures the realization of three representative configurations of the effective potential, we will point out a few properties of $V_{\text {tot }}$ that are strictly related to the peculiar form of the action (2) which generates the one-loop effective potential in Eq. (18).

The first issue concerns the behaviour of $V_{\text {tot }}$ at large values of the field. We notice that, for a generic tree potential (i.e. not for the particular potential in Eq. (19)) whose leading term at large $\phi$ is $\left(g_{q} / q\right.$ !) $\phi^{q}$ (with $g_{q}>0$ ), the power $q$ must fulfill the condition

$q=\frac{5}{4}(q-2)$

i.e. $q=10$, in order to balance the tree potential and the quantum corrections in Eq. (18), that appear with differ- 
ent sign. If $q>10, V_{\text {tot }}$ goes negative at large $\phi$, thus showing instability; if $q<10, V_{\text {tot }}$ grows positive at large $\phi$, while if $q=10$ one has the critical value $g_{10} \mathrm{cr}=$ $4480 \pi^{10} \alpha^{6} /\left(729 \Gamma(3 / 4)^{8}\right) \approx 1.13 \times 10^{5} \alpha^{6}$ above which the effective potential is negative and below which it is positive. At $g_{10}=g_{10_{\mathrm{cr}}}$, the large field behavior of the potential is ruled by powers of the field that are smaller than 10 , and therefore it is positive. Remarkably, as indicated in Eq. (3), $\phi^{10}$ corresponds to the marginally scaling operator according to the Lifshitz scaling.

Now we investigate on the condition of masslessness of the effective potential at $\phi=0$, which is specified by the condition (the subscript 0 means that it is evaluated at $\phi=0$ )

$m_{0 \mathrm{eff}}^{2}=\left.\frac{d^{2} V_{\mathrm{tot}}}{d \phi^{2}}\right|_{\phi=0}=0$

This issue is strictly related to the presence of a non-trivial minimum at $\phi \neq 0$, as it is clear that a negative curvature (mass square parameter) at $\phi=0$ for a well-behaved effective potential that diverges positively at large $\phi$, indicates the presence of minima at $\phi \neq 0$.

We start by considering a simple monomial tree potential $V=\left(g_{q} / q !\right) \phi^{q}$ with $q>2$, so that we assume for the moment a zero tree mass in our problem. By looking at the structure of $V_{\text {tot }}$ in Eq. (18), we immediately realize that we get $m_{0}{ }_{\text {eff }}^{2} \neq 0$ only if $(5 / 4)(q-2)=2$, that selects the particular value $q=18 / 5$. Instead, if we take a monomial potential with $q>18 / 5$, we find $m_{0}^{2} 2=0$ and, if $q<18 / 5$, the curvature at the origin diverges. If we limit ourselves to integer $q$, then the first value that does not generate singularities at the origin is $q=4$. In general, integer $q$, with $4 \leqslant q \leqslant 10$ generate regular effective potentials with $m_{0}^{2}$ eff $=0$.

We go one step further and search for non-trivial minima of the effective potential in the case of monomial tree potential $V=\left(g_{q} / q !\right) \phi^{q}$ and, with the help of Eq. (18), we find in this case

$V_{\mathrm{tot}}=\frac{g_{q}}{q !} \phi^{q}-\gamma\left(\frac{g_{q}}{(q-2) !} \phi^{q-2}\right)^{5 / 4}$,

where, for the sake of simplicity, we defined

$\gamma=\frac{\Gamma\left(\frac{3}{4}\right)^{2}}{10 \pi^{5 / 2} \alpha^{3 / 2}}$

and the extremum condition $V_{\text {tot }}^{\prime}=0$ admits, besides $\phi=0$, the non-zero solution

$\phi_{\min }=\left(\frac{5(q-1) ! \gamma g_{q}^{1 / 4}}{4((q-2) !)^{1 / 4}(q-3) !}\right)^{\frac{4}{10-q}}$ provided that $4 \leqslant q<10$. This solution, as discussed above, must be a minimum, in opposition to the maximum at the origin. The negative value of the effective potential at $\phi_{\min }$ can be straightforwardly computed and, in particular, we display the results for $q=4$

$\phi_{\min }=\left(\frac{15 \gamma g_{4}^{1 / 4}}{2^{5 / 4}}\right)^{\frac{2}{3}}=\left(\frac{3 \Gamma\left(\frac{3}{4}\right)^{2} g_{4}^{1 / 4}}{2^{9 / 4} \pi^{5 / 2} \alpha^{3 / 2}}\right)^{\frac{2}{3}}$

and

$V_{\text {tot }}\left(\phi_{\min }\right)=-\frac{455^{2 / 3} 3^{2 / 3}}{322^{1 / 3}} g_{4}^{\frac{5}{3}} \gamma^{\frac{8}{3}}$

Therefore the structure of Eq. (18) with a monomial tree potential with $4 \leqslant q<10$ and vanishing tree mass, $m^{2}=0$, yields a double well effective potential with zero curvature at the origin. One should be aware that this picture is somehow different from the one of the Coleman-Weinberg one-loop scalar potential, where the only dimensionful scale is generated by the radiative corrections, while in our case (even when $q=4$ and the coupling $g_{4}$ is dimensionless) we start with at least one dimensionful input parameter, namely $\alpha$.

At this point we go back to the study of the curvature of the effective potential at $\phi=0$, but now we allow for a finite tree mass term, i.e. we take the tree potential as

$V=\frac{1}{2} m^{2} \phi^{2}+\frac{g_{q}}{q !} \phi^{q}$

with $m^{2}>0$, and expand the second derivative of the effective potential in Eq. (18) as

$m_{0 \text { eff }}^{2}=\left.\frac{d^{2} V_{\mathrm{tot}}}{d \phi^{2}}\right|_{\phi=0}=m^{2}-\gamma\left(\frac{5}{16 m^{3 / 2}} \lim _{\phi \rightarrow 0} V^{\prime \prime \prime 2}+\frac{5 m^{1 / 2}}{4} \lim _{\phi \rightarrow 0} V^{\prime \prime \prime \prime}\right)$

Then, if $2<q<3$ or $3<q<4$, a singularity shows up in the right hand side of Eq. (29) and therefore, in order to have a regular effective potential, we must take either $q=3$ or $q \geqslant 4$. In addition, when $q=3$ and $q=4$, there is a finite quantum correction to $m^{2}$ while, if $q>4$, the correction vanishes and $m_{0}^{2} 2=m^{2}$. For $q=3$ we get

$m_{0 \mathrm{eff}}^{2}=m^{2}-\frac{\Gamma\left(\frac{3}{4}\right)^{2}}{32 \pi^{5 / 2} \alpha^{3 / 2}} \frac{g_{3}^{2}}{m^{3 / 2}}$

but, as we are mainly interested in even potentials, we do not further analyze the case $q=3$ and we look instead at $q=4$, where we get, according to the definition (24),

$m_{0}^{2}=m^{2}-\frac{\Gamma\left(\frac{3}{4}\right)^{2}}{8 \pi^{5 / 2} \alpha^{3 / 2}} m^{1 / 2} g_{4}=m^{2}-\frac{5 \gamma m^{1 / 2} g_{4}}{4}$. 
Equation (31) shows that the sign of $m_{0}^{2}$ iff determined by the relative size of the parameters $m^{2}, \alpha$ and $g_{4}$. In particular, by defining

$C\left(m^{2}\right)=\frac{5 \gamma g_{4}}{4 m^{3 / 2}}$

we find that $m_{0}^{2}$ eff $=0$ occurs both at $m^{2}=0$ and at $m^{2}=$ $\bar{m}^{2}$, where $\bar{m}^{2}$ is such that $C\left(\bar{m}^{2}\right)=1$.

For large positive $m^{2}$, the tree mass is dominant in Eq. (31) and therefore $m_{0}^{2}$ eff $^{2}>0$ and it corresponds to $0<C<1$. Conversely, for small positive $m^{2}$, i.e. $C>1$, the negative quantum correction is dominant in Eq. (31) and we have $m_{0}^{2}{ }_{\text {eff }}^{2}<0$.

Therefore, for large $m^{2}(0<C<1)$ the curvature at the origin, as expected, is positive (a particular example of this configuration is given in Fig. 1) but, more interestingly, we find a full interval of $m^{2}$, from $m^{2}=0(C \rightarrow \infty)$ to the critical value $\bar{m}^{2}$ associated to $C=1$, that yields negative curvatures at $\phi=0$ (with vanishing curvature at the two endpoints of the interval) and consequently implies the onset of a new couple of minima at $\phi \neq 0$.

In fact, the case with $m^{2}=0$ has been discussed before in the context of monomial potentials and the corresponding minima associated to SSB have been determined. When $m^{2}$ grows from zero, the curvature at the origin diminishes from zero and becomes negative, and the corresponding SSB minima can be determined numerically (Fig. 2 is an example of this kind). The location of these minima approaches zero when $m^{2} \rightarrow \bar{m}^{2}$ and they disappear for $m^{2}>\bar{m}^{2}$, which marks the transition to the symmetric phase with the only minimum of the effective potential located at $\phi=0$.

In summary, the picture observed for this Lifshitz-type action is rather different from that of the simple scalar effective potential, as in the former case there is a finite range of values of $m^{2}>0$ that produces negative curvature at $\phi=0$ and SSB with non-trivial minima, while in the latter case the negative curvature is obtained only for $m^{2}<0$. In addition, in the former case there are two different values, namely $m^{2}=0$ and $m^{2}=\bar{m}^{2}$, associated to zero curvature $m_{0}^{2}$ eff $^{2}=0$. As already noticed, these differences are essentially due to the presence of more than one dimensionful parameter in our problem that give origin to a richer structure.

Finally, the case with $m^{2}<0$, that corresponds to a SSB tree level potential, presents the problem of complex quantum corrections (at least for small values of the field $\phi$ ), due to the term $V^{\prime \prime 5 / 4}$ in Eq. (18). In this case, as expected, a couple of minima at $\phi \neq 0$ is always present and $V_{\text {tot }}\left(\phi_{\min }\right)$ has zero imaginary part. In our analysis, we focused only on the real part of $V_{\text {tot }}$ and a specific example of this kind is displayed in Fig. 3.

\section{Flow of the effective potential and beta-functions}

After analysing the renormalized one-loop effective potential, we now turn to the issue of determining the flow equation for the scale-dependent potential. To this purpose, we recall that the standard procedure is obtained by applying $k_{\mathrm{IR}} d / d k_{\mathrm{IR}}$ to the effective potential regulated by an infrared scale $k_{\mathrm{IR}}$ and, in our case, it is easy to realize that this is equivalent to applying $-k d / d k$ to Eq. (14), properly regularized in accordance to Eq. (15), and the factor $(-1)$ is introduced to compensate the exchange of the UV and IR cutoff. Therefore, we get

$$
\begin{aligned}
k \frac{d}{d k} V_{11}(k)= & -\frac{V^{\prime \prime 9 / 8}}{4 \sqrt[4]{2} \pi^{3 / 2} \alpha^{9 / 4}} \frac{k^{1 / 2}}{B^{1 / 4}}\left[2 \alpha \Gamma\left(\frac{7}{4}\right)\right. \\
& \times\left(I_{-\frac{9}{4}}\left(\frac{B \sqrt{V^{\prime \prime}}}{\alpha k^{2}}\right)+2 I_{\frac{9}{4}}\left(\frac{B \sqrt{V^{\prime \prime}}}{\alpha k^{2}}\right)\right) \\
& +\frac{B}{k^{2}} \Gamma\left(\frac{3}{4}\right) \sqrt{V^{\prime \prime}}\left(I_{\frac{13}{4}}\left(\frac{B \sqrt{V^{\prime \prime}}}{\alpha k^{2}}\right)\right. \\
& \left.\left.-I_{-\frac{5}{4}}\left(\frac{B \sqrt{V^{\prime \prime}}}{\alpha k^{2}}\right)\right)\right] .
\end{aligned}
$$

From the above expression one could get the naive expectation that the flow of the potential is always proportional to the square root $\sqrt{k}$. However, this is not true, because, for example, in the large $k$ regime we also have to expand in series the dependence on $k$ in the argument $x$ of the Bessel functions. It is known that when $x \rightarrow 0$, one has

$$
I_{b}(x) \underset{x \rightarrow 0}{\sim} \frac{1}{\Gamma(1+b)}\left(\frac{x}{2}\right)^{b}+O\left(x^{b+2}\right)
$$

and Eq. (34) allows to establish the UV regime $(x=$ $\left.B \sqrt{V^{\prime \prime}} /\left(\alpha k^{2}\right) \rightarrow 0\right)$ of the RG flow of the potential, where it is assumed $k \gg \alpha^{-1}$ and only the two leading powers of $k$ are retained:

$k \frac{d}{d k} V_{11}(k) \underset{\overline{U V}}{=}-\frac{15}{32 \pi^{3 / 2}} \frac{\alpha}{B^{5 / 2}} k^{5}-\frac{V^{\prime \prime} k}{32 \pi^{3 / 2} \alpha \sqrt{B}}+O\left(k^{-3 / 2}\right)$.

The flow in the IR regime can be obtained similarly:

$k \frac{d}{d k} V_{11}(k) \underset{I R}{=}-\frac{\Gamma\left(\frac{3}{4}\right) B^{1 / 4} V^{\prime \prime 11 / 8} \exp \left(-\frac{B \sqrt{V^{\prime \prime}}}{\alpha k^{2}}\right)}{4 \pi^{2} \sqrt[4]{2} \alpha^{7 / 4} \sqrt{k}}$.

This flow is a non-analytic function of $k$ and goes to zero like $\exp \left(-1 / k^{2}\right)$, so that the running is exponentially dumped at low momenta.

Now, we derive the $\beta$-functions of some relevant couplings from Eq. (33). The couplings are defined by the 
parametrization of potential in (3) and this time, for illustrative purposes, we focus on the first three parameters, namely $m^{2}(k), \lambda(k) \equiv \lambda_{1}(k)$ and $g(k) \equiv \lambda_{2}(k)$. Their $\beta$-functions are obtained by projecting the flow of the potential onto the specific subspace of each coupling through successive differentiation of the right hand side of Eq. (33) with respect to the field.

Then, for the square mass parameter we find $(w \equiv$ $\left.(B m) /\left(\alpha k^{2}\right)\right)$

$$
\begin{aligned}
\beta_{m^{2}}= & \frac{\lambda B^{\frac{7}{4}}}{32 \pi^{\frac{3}{2}} \alpha^{\frac{13}{4}} k^{\frac{7}{2}}}\left(\frac{m}{2}\right)^{\frac{1}{4}}\left[2 \Gamma\left(\frac{3}{4}\right)\right. \\
& \times\left(\frac{27 \alpha^{2} k^{4}}{B^{2}}+2 m^{2}\right) I_{-\frac{9}{4}}(w)+m \Gamma\left(-\frac{1}{4}\right) \\
& \left.\times\left(\frac{3 \alpha k^{2}}{B}\left(I_{\frac{5}{4}}(w)-I_{-\frac{13}{4}}(w)\right)+m I_{\frac{9}{4}}(w)\right)\right] \\
\overline{U V} & -\frac{k \lambda}{32 \pi^{\frac{3}{2}} \alpha \sqrt{B}},
\end{aligned}
$$

where the right hand side shows the result obtained in the $\mathrm{UV}$ regime, i.e. with $k \gg \alpha^{-1}, m$. In fact, in this limit many irrelevant details associated to the particular nature of the IR regulator become negligible. We observe that $\beta_{m^{2}}$ is linearly proportional to the quartic coupling $\lambda$.

We also notice that $\beta_{m^{2}}$ corresponds to a dimensionful parameter $\left(\left[\mathrm{m}^{2}\right]=2\right)$, while the $\beta$-function of the associated dimensionless coupling $\tilde{m}^{2}=m^{2} B / k^{2}$ is: $\beta_{\tilde{m}^{2}}=$ $-2 \tilde{m}^{2}+B k^{-2} \beta_{m^{2}}$, where the first term is generated from the dimension of the original coupling $m^{2}$.

Similarly, one derives $\beta_{\lambda}$ of the dimensionless quartic coupling $\lambda$, displayed below only in its simplified form in the UV regime. It turns out to be proportional to the sextic coupling $g \equiv \lambda_{2}$ :

$\beta_{\lambda} \underset{U V}{=}-\frac{g k}{32 \pi^{\frac{3}{2}} \alpha \sqrt{B}}=-\frac{\tilde{g}}{32 \pi^{\frac{3}{2}} \tilde{\alpha}}$

and, in the right hand side of (38), $\beta_{\lambda}$ is expressed in terms of dimensionless variables $\tilde{g}=g k^{2} / B$ and $\tilde{\alpha}=\alpha k / \sqrt{B}$. The same procedure, carried out for the other relevant couplings, shows again that $\beta_{\lambda_{2}} \propto \lambda_{3}$ and $\beta_{\lambda_{3}} \propto \lambda_{4}$.

Note that we retained the factor $B$, introduced in Eq. (15), into the definition of running scale and in the consequent definition of the above dimensionless variables, as $B$ is an indispensable element in the rescaling procedure. Then, only within this scheme and in the UV limit, one recovers universal expressions (i.e. independent of the details of the IR regulator, such as $B$ ) for the one-loop $\beta$-functions of the dimensionless couplings.

\section{Conclusions}

In conclusion, we developed a new regularization scheme, suitable for studying the physics around anisotropic Lifshitz points, by a standard treatment of the time coordinate and by adapting the proper time regulator to the three-dimensional subset of space coordinates, by means of the integral representation of the square root in (7). Then, the UV divergences are regulated by a sharp cutoff on the proper time variable $s$. With the help of this scheme, we computed the one-loop effective potential, by determining the correct counterterms to get finite quantum corrections, which turn out to decrease the value of the tree potential at each value of the field $\phi$, as shown in the three figures. We also pointed out that the presence in our problem of two or more dimensionful scales gives origin to a rich structure of the phase diagram, which allows for a full interval of positive values of the tree level square mass that are associated to a negative curvature at the origin, with consequent onset of SSB at the one-loop level.

Then, from the dependence of the potential and its couplings on the cutoff scale, we derived a flow equation for the effective potential and the $\beta$-functions for the couplings $m^{2}$ and $\lambda$, and we found that $\beta_{\lambda_{n}}$ is proportional to the subsequent coupling $\lambda_{n+1}$, in agreement with the findings of [8].

The $\beta$-functions, are rather different from those calculated for the theory in proximity of the Gaussian fixed point, because they are a consequence of the nature of the ultraviolet divergences associated to a Lifshitz point. In fact, an inspection of the diagrams within the Lifshitz scaling indicates only one divergent one-loop diagram for each $(2 n+2)$ point Green's function, namely the tadpole generated by the coupling $\lambda_{n+1}$.

Moreover, a comparison with the counterterms determined in [8], allows us to select a particular value of the parameter $B$, i.e. the proportionality constant between the proper time cutoff $s_{\mathrm{UV}}$ and the inverse square running scale $k^{-2}$. Actually, by choosing $\sqrt{B}=\sqrt{\pi} / 4$ in (37) and (38), we get, $\beta_{m^{2}}=-k \lambda /\left(8 \pi^{2} \alpha\right), \beta_{\lambda}=-k g /\left(8 \pi^{2} \alpha\right)$, that reproduce the results obtained with a sharp 3-momentum UV cutoff in [8].

Acknowledgements $\mathrm{AB}$ thanks Ugo Moschella for important comments. MP was supported by a KIAS Individual Grant (PG062001) at Korea Institute for Advanced Study and by Basic Science Research Program through the National Research Foundation of Korea funded by the Ministry of Education (NRF-2016R1D1A1B03933399). The work of LR was partially supported by the ACRI-INFN Research Award within Young Investigator Training Program 2018 in the project "Functional and Renormalization-Group Methods in Quantum and Statistical Physics". MP and LR would like to thank INAF-Catania Astrophysical Observatory for kind hospitality.

Data Availability Statement This manuscript has no associated data or the data will not be deposited. [Authors' comment: This is a theoretical study and no experimental data has been listed.] 
Open Access This article is licensed under a Creative Commons Attribution 4.0 International License, which permits use, sharing, adaptation, distribution and reproduction in any medium or format, as long as you give appropriate credit to the original author(s) and the source, provide a link to the Creative Commons licence, and indicate if changes were made. The images or other third party material in this article are included in the article's Creative Commons licence, unless indicated otherwise in a credit line to the material. If material is not included in the article's Creative Commons licence and your intended use is not permitted by statutory regulation or exceeds the permitted use, you will need to obtain permission directly from the copyright holder. To view a copy of this licence, visit http://creativecomm ons.org/licenses/by/4.0/.

Funded by SCOAP ${ }^{3}$.

\section{References}

1. R. Hornreich, M. Luban, S. Shtrikman, Critical behavior at the onset of k-space instability on the $\lambda$ line. Phys. Rev. Lett. 35, 16781681 (1975)

2. R.M. Hornreich, The Lifshitz point: phase diagrams and critical behavior. J. Magn. Magn. Mater. 15, 387-392 (1980)

3. W. Selke, The ANNNI model, theoretical analysis and experimental application. Phys. Rep. 170, 213-264 (1988)

4. H. Diehl, Critical behavior at M-axial Lifshitz points. Acta Phys. Slov. 52, 271-283 (2002)

5. P. Horava, Quantum criticality and Yang-Mills gauge theory. Phys. Lett. B 694, 172-176 (2011). arXiv:0811.2217

6. R. Iengo, J.G. Russo, M. Serone, Renormalization group in Lifshitz-type theories. JHEP 11, 020 (2009). arXiv:0906.3477

7. K. Kikuchi, Restoration of Lorentz symmetry for Lifshitz type scalar theory. Prog. Theor. Phys. 127, 409-431 (2012). arXiv: 1111.6075

8. M. Eune, W. Kim, E.J. Son, Effective potentials in the Lifshitz scalar field theory. Phys. Lett. B 703, 100-105 (2011). arXiv: 1105.5194

9. P. Horava, Quantum gravity at a Lifshitz point. Phys. Rev. D 79, 084008 (2009). arXiv:0901.3775

10. R.-G. Cai, L.-M. Cao, N. Ohta, Thermodynamics of black holes in Horava-Lifshitz gravity. Phys. Lett. B 679, 504-509 (2009). arXiv:0905.0751

11. M. Eune, W. Kim, Lifshitz scalar, brick wall method, and GUP in Horava-Lifshitz gravity. Phys. Rev. D 82, 124048 (2010). arXiv: 1007.1824

12. R. Brandenberger, Matter bounce in Horava-Lifshitz cosmology. Phys. Rev. D 80, 043516 (2009). arXiv:0904.2835

13. E.J. Son, W. Kim, Smooth cosmological phase transition in the Horava-Lifshitz gravity. JCAP 06, 025 (2010). arXiv:1003.3055
14. H. Diehl, M. Shpot, Critical, crossover, and correction to scaling exponents for isotropic Lifshitz points to order $(8-\mathrm{d})^{* * 2}$. J. Phys. A35, 6249-6260 (2002). arXiv:cond-mat/0204267

15. A. Bonanno, D. Zappala, Isotropic Lifshitz critical behavior from the functional renormalization group. Nucl. Phys. B 893, 501-511 (2015). arXiv: 1412.7046

16. D. Zappala, Isotropic Lifshitz point in the $\mathrm{O}(\mathrm{N})$ theory. Phys. Lett. B 773, 213-218 (2017). arXiv:1703.00791

17. D. Zappala, Indications of isotropic Lifshitz points in four dimensions. Phys. Rev. D 98(8), 085005 (2018). arXiv:1806.00043

18. D. Zappala, Isotropic Lifshitz scaling in four dimensions. Int. J. Geom. Methods Mod. Phys. 17(04), 2050053 (2020). arXiv:1912.03071

19. N. Defenu, A. Trombettoni, D. Zappala, Topological phase transitions in four dimensions. arXiv:2003.04909

20. J.S. Schwinger, On gauge invariance and vacuum polarization. Phys. Rev. 82, 664-679 (1951)

21. M. Oleszczuk, A symmetry preserving cutoff regularization. Z. Phys. C 64, 533-538 (1994)

22. S.-B. Liao, On connection between momentum cutoff and the proper time regularizations. Phys. Rev. D 53, 2020-2036 (1996). arXiv:hep-th/9501124

23. S.-B. Liao, Operator cutoff regularization and renormalization group in Yang-Mills theory. Phys. Rev. D 56, 5008-5033 (1997). arXiv:hep-th/9511046

24. O. Bohr, B. Schaefer, J. Wambach, Renormalization group flow equations and the phase transition in $\mathrm{O}(\mathrm{N})$ models. Int. J. Mod. Phys. A 16, 3823-3852 (2001). arXiv:hep-ph/0007098

25. A. Bonanno, D. Zappalà, Towards an accurate determination of the critical exponents with the renormalization group flow equations. Phys. Lett. B 504, 181-187 (2001). arXiv:hep-th/0010095

26. D.F. Litim, J.M. Pawlowski, Completeness and consistency of renormalisation group flows. Phys. Rev. D 66, 025030 (2002). arXiv:hep-th/0202188

27. D.F. Litim, J.M. Pawlowski, Wilsonian flows and background fields. Phys. Lett. B 546, 279-286 (2002). arXiv:hep-th/0208216

28. A. Bonanno, G. Lacagnina, Spontaneous symmetry breaking and proper-time flow equations. Nucl. Phys. B 693, 36-50 (2004). arXiv:hep-th/0403176

29. S.P. de Alwis, Exact RG flow equations and quantum gravity. J. High Energy Phys. 3, 118 (2018). arXiv: 1707.09298

30. A. Bonanno, S. Lippoldt, R. Percacci, G.P. Vacca, On exact proper time Wilsonian RG flows. Eur. Phys. J. C 80(3), 249 (2020). arXiv:1912.08135

31. C. Farias, M. Gomes, J. Nascimento, A. Petrov, A. da Silva, On the effective potential for Horava-Lifshitz-like theories. Phys. Rev. D 85, 127701 (2012). arXiv:1112.2081 